\title{
Investigation of beam echo generation with electrons in
}

\section{the IOTA ring}

\author{
Annika Gabriel, Case Western Reserve University, Cleveland, OH 44106 \\ Dr. Tanaji Sen, Accelerator Division, Fermilab, Batavia, IL 60510 \\ Professor Michael Martens, Department of Physics, Case Western Reserve \\ University, Cleveland, $\mathrm{OH} 44106$
}

July 20, 2019

\begin{abstract}
This project investigates the possibility of echo generation with electrons in the IOTA ring. Through simulations the effects of different beam and ring parameters on echo amplitude and full width half max are tested. We find that transverse coupling and delay time have significant effects on echo generation and will have to be carefully controlled in future experiments. Effect of decoherence time, quadrupole kick strength, quantum excitation, synchrotron damping, and momentum spread are also investigated. It is found that it is possible to produce echoes of significant amplitude and full width half max in the IOTA ring despite the effects of synchrotron damping. Relation and agreement of echoes produced with predictions of nonlinear theory of beam echoes is also investigated. Findings from this study can be used to inform future beam echo experiments at the IOTA ring.
\end{abstract}




\section{Background}

\subsection{Beam Echoes}

Echoes are a common phenomenon in physics. Echoes have been observed in a variety of systems such as with spin, photons, plasma waves, and systems of ultracold atoms [6]. Many of these echo phenomena have since been developed into diagnostic tools $[6]$. For example, spin echoes, which were discovered by Hahn, are now used as diagnostic tools in nuclear magnetic resonance imaging. Echoes were first introduced to the field of accelerator physics around two decades ago and have since been observed at the Fermilab Antiproton Accumulator and at the CERN Super Proton Synchrotron [6]. The most detailed studies of beam echoes to date have been carried out at the Relativistic Heavy Ion Collider where an analysis of experiments was performed to extract diffusion coefficients [5]. In this project we seek to expand the current understanding of beam echoes by determining if they can be produced with electrons in the Integrable Optics Testing Accelerator (IOTA) at Fermilab.

A beam echo is a recoherence in phase space (with position and momentum as the phase space coordinates) of a bunch of particles in an accelerator that had previously undergone decoherence. In this context a coherent beam is defined as a beam where all particles are oscillating with the same frequency and phase. A beam decoheres when its particles fall out of phase and can no longer provide a coherent beam. An echo is initiated by first delivering a dipole kick to the beam which moves the beam centroid off the closed orbit to a larger amplitude. There the particles at different amplitudes execute betatron oscillations with different frequencies due to the nonlinear elements in the lattice. The lattice refers to the configuration of magnets that make up the main components of the accelerator. The different frequencies cause the particles to go out of phase and the centroid decays back towards the closed orbit. The time taken (called $t_{D}$ ) for the centroid amplitude to fall to $1 / e$ of its initial amplitude following the dipole kick characterizes the decoherence. Once the beam has completely decohered, typically by time $2 t_{D}$, and the centroid is back on the 
closed orbit, a quadrupole kick is applied to the beam at a time $\tau>2 t_{D}$ after the dipole kick.

The quadrupole kick does not affect the beam centroid or amplitude so the average centroid position will remain zero. The echo occurs after another time interval $\tau$ has passed. At time interval $\tau$ after the quadrupole kick is applied the beam signal will suddenly and briefly recohere, producing a pronounced blip. This recoherence is due to "the correlation and interplay between the two kicks and a long memory of the intricate beam dynamics in phase space" [1]. This blip is referred to as the beam echo and is the phenomenon that this project has produced and studied in the IOTA ring with electrons. An example of a beam echo is shown in 1 below.

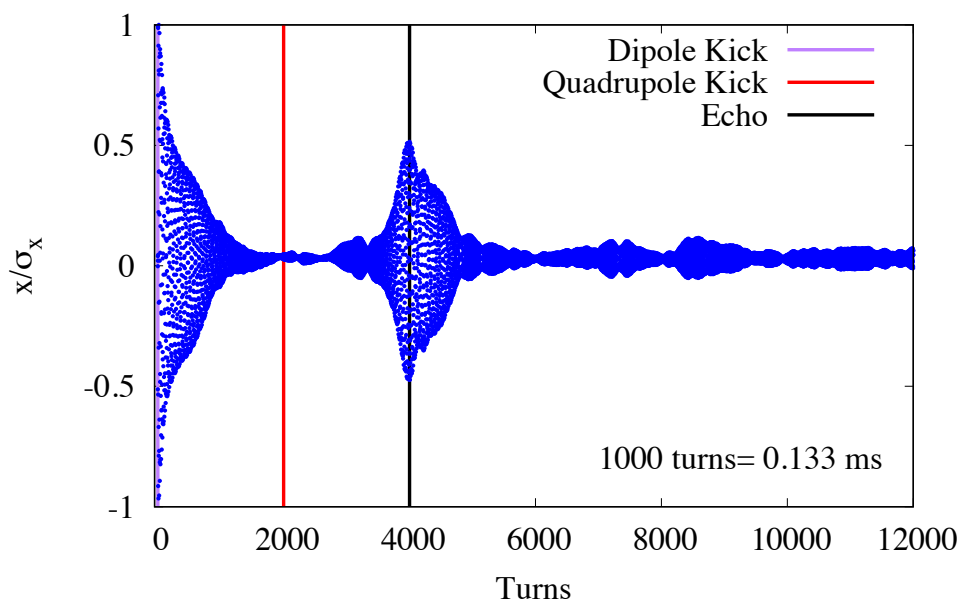

Figure 1: An example of a beam echo showing dipole kick and quadrupole kick. The xaxis "Turns" are completed revolutions around the ring and the y-axis is the relative beam oscillation amplitude in the $\mathrm{x}$ direction.

Beam echoes are "exquisitely sensitive" [6] to beam diffusion. Beam diffusion is a consequence of intensity, space charge, beam-beam interaction, and many other effects. Diffusion in accelerators is detrimental because it can lead to emittance growth, formation of "beam halo", and particle loss. All of these effects degrade the performance of the accelerator. Limiting diffusion is an essential step in improving accelerator performance. However, the standard method to measure transverse diffusion (beam scraping) is costly, time consuming, and impractical in many accelerator applications. Beam scraping takes several hours to 
complete and thus is only applicable to storage rings that can hold a beam for the necessary amount of time. Beam echoes can be generated on the order of milliseconds and represent a novel method to measure transverse diffusion. Beam echoes could allow diffusion to be measured in synchrotrons where beams circulate for relatively short times [2]. Improved measurement of diffusion can lead to a better understanding of what causes it and how to reduce its effects.

\subsection{Previous Work and Motivation}

Similar research to the work proposed for this project has been conducted with protons. A summer intern of Dr. Tanaji Sen, Dhruv Desai, studied the generation of beam echoes with protons in the IOTA ring [2]. His results showed that multiple echoes could be produced in simulations of the IOTA ring and that they were robust to small errors in the lattice [2]. In this project we hope to expand the methods used to generate echoes with electrons. Showing the ability to generate echoes with electrons would represent a novel development because electrons possess several properties that make it much more difficult to produce an echo. Currently it is generally thought that beam echoes cannot be produced with electron beams or in electron storage rings. Because of this there is a lack of knowledge about beam echo generation with electrons and their relation to various accelerator parameters.

It is difficult to generate a beam echo with electrons because synchrotron radiation for electrons is much greater than that for protons. Electrons generate synchrotron radiation due to centripetal acceleration in the accelerator, and in the process they produce photons. This photon production causes the electrons to lose energy which is replenished by the RF cavities but also results in beam damping. This damping of transverse oscillations makes it difficult to observe echoes. In addition, synchrotron radiation causes diffusion in the beam and large diffusion can suppress the echo effect entirely. These difficulties have so far made it impossible to generate echoes in electron beams. In this paper it is shown that it is possible to generate beam echoes with electrons in the IOTA ring despite these difficulties. Using 
the results of this research the benefits of improved diffusion measurement with beam echoes could be extended to new applications such as electron storage rings.

\subsection{IOTA: Integrable Optics Testing Accelerator}

IOTA is "a storage ring for advanced high intensity beam physics research" at Fermilab's Accelerator Science and Technology (FAST) facility [4]. It is a compact ring for accelerating both electrons and protons. The IOTA research program focuses on "the study of nonlinear focusing integrable optical beam lattices based on special magnets and electron lenses, beam dynamics of space-charge effects and their compensation, optical stochastic cooling, and several other experiments" [4]. A schematic of the IOTA ring can be seen in 2 and a table of general IOTA parameters for operation with electrons can be seen in 3 .

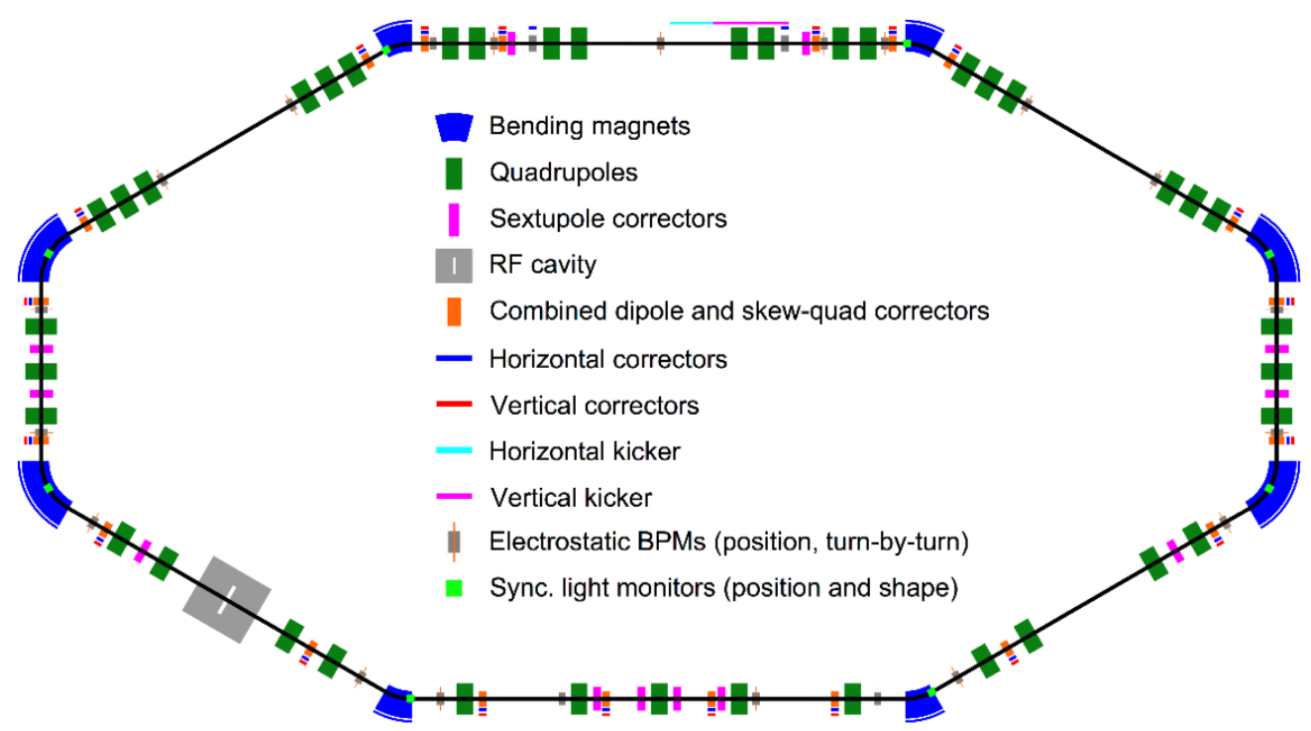

Figure 2: Diagram of layout of main IOTA ring components [4]. 


\begin{tabular}{|l|l|}
\hline Parameter & Value \\
\hline Beam energy, $E$ & $150 \mathrm{MeV}$ \\
\hline Circumference, revolution period, $C_{0}, T_{0}$ & $39.97 \mathrm{~m}, 133.3 \mathrm{~ns}$ \\
\hline Betatron tunes, $Q_{x}, Q_{y}$ & $4-6$ \\
\hline Maximum beta-function, $\beta_{x}, \beta_{y}$ & $8.5,4 \mathrm{~m}$ \\
\hline Momentum compaction, $\alpha_{p}$ & 0.067 \\
\hline RF voltage, frequency, revolution harmonic & $1 \mathrm{kV}, 30.0 \mathrm{MHz}, 4$ \\
\hline Synchrotron tune, $Q_{s}$ & $5.3 \times 10^{-4}$ \\
\hline Number of particles, beam current, $N_{e}, I_{e}$ & $2 \times 10^{9}, 2.4 \mathrm{~mA}$ \\
\hline Equilibrium beam emittance, $\varepsilon_{x}, \varepsilon_{y}$ & $0.04,0.04 \mu \mathrm{m}$ \\
\hline Beam energy spread, bunch length, $\sigma_{E}, \sigma_{z}$ & $1.35 \times 10^{-4}, 10.8 \mathrm{~cm}$ \\
\hline Radiation damping times, $\tau_{x}, \tau_{y}, \tau_{z}$ & $0.9 \mathrm{~s}, 0.9 \mathrm{~s}, 0.24 \mathrm{~s}$ \\
\hline
\end{tabular}

Figure 3: Table of relevant IOTA parameters for operation with electrons [4].

IOTA presents a unique opportunity to study beam echoes, and specifically beam echoes produced with electrons. Almost all previous echo research that has been conducted has focused on echo generation with protons or heavy ions. It is commonly thought that the "echo effect is not relevant to electron storage rings" [1] because of very strong diffusion and radiation damping caused by synchrotron radiation. The transverse radiation damping time is defined as the time necessary for a beam's energy to be reduced by $1 / e$ of its original value. The radiation damping time in turns (revolutions around the accelerator ring) can be calculated using the following equations and parameters [7].

$I_{2}$ and $I_{4}$ are called the 2 nd and 4 th synchrotron radiation integrals and are thought of as general parameters of the accelerator lattice. They are dependent on the average bending radius of the bending magnets in the lattice $(\rho)$, the horizontal dispersion $\left(\eta_{x}\right)$, the reference particle momentum $\left(P_{O}\right)$, and the magnetic field $(\mathrm{B})$.

$$
\begin{array}{r}
I_{2}=\oint \frac{1}{\rho^{2}} d s \\
I_{4}=\oint \frac{\eta_{x}}{\rho}\left(\frac{1}{\rho^{2}}+2 k_{1}\right) d s \\
k_{1}=\frac{e}{P_{o}} \frac{\delta B_{y}}{\delta x}
\end{array}
$$

The sychrotron radiation integrals can be used to calculate the horizontal damping partition 
number $j_{x}$, and the reference energy of the beam $\left(E_{o}\right)$, and the energy lost per turn of the accelerator $\left(U_{o}\right)$.

$$
\begin{array}{r}
j_{x}=2+\frac{I_{4}}{I_{2}} \\
U_{o}=\frac{C_{\gamma}}{2 \pi} E_{o}^{4} I_{2}
\end{array}
$$

Where $C_{\gamma}$ is a physical constant given by:

$$
C_{\gamma}=\frac{e^{2}}{\left.3 \epsilon_{o}\left(m c^{2}\right)^{4}\right)} \approx 8.846 \times 10^{-5} \mathrm{~m} / G e V^{3}
$$

Using these variables along with the time to complete one revolution $\left(T_{o}\right)$ it is possible to calculate the transverse radiation damping time $\tau_{x}$.

$$
\tau_{x}=\frac{2 E_{o} T_{o}}{j_{x} U_{o}}
$$

The transverse damping time for IOTA is found to be $0.9 \mathrm{~s}$ or approximately $6.8 \times 10^{6}$ turns [4]. In most synchrotron storage rings radiation damping overpowers the echo effect and makes it impossible to produce. But IOTA has a transverse damping time that is much longer than the few thousand turns necessary to generate a beam echo. Because of this it is possible in IOTA to produce an echo with electrons despite the effect of damping.

\section{Methods}

The MAD-X (Methodical Accelerator Design) program is a general-purpose tool for chargedparticle optics design and studies in alternating-gradient accelerators and beam lines [3]. In order to achieve the objectives of this project, simulations will be carried out in the MADX simulation program. These simulations can be used to track particles throughout 
the accelerator, edit and add accelerator elements, determine particle and beam properties, and optimize accelerator performance. A simulation lattice of the IOTA ring has already been constructed by the designers of the ring at Fermilab. This lattice will be used and edited to determine the possibility of echo generation and relevant parameters of the IOTA ring. Analysis of simulation data was carried out using $\mathrm{C}++$ and Python in order to determine the effect of various accelerator parameters on echo generation. In addition, theoretical calculations and predictions from the non-linear theory are carried out in Mathematica. These calculations will be used to compare simulation results to predictions from non-linear theory

\section{Results}

\subsection{Dipole Kick And Decoherence Time}

The first step in echo production is application of the dipole kick in order to achieve beam decoherence. It is important to determine beam decoherence time so the optimal time for application of the quadrupole kick can be determined.
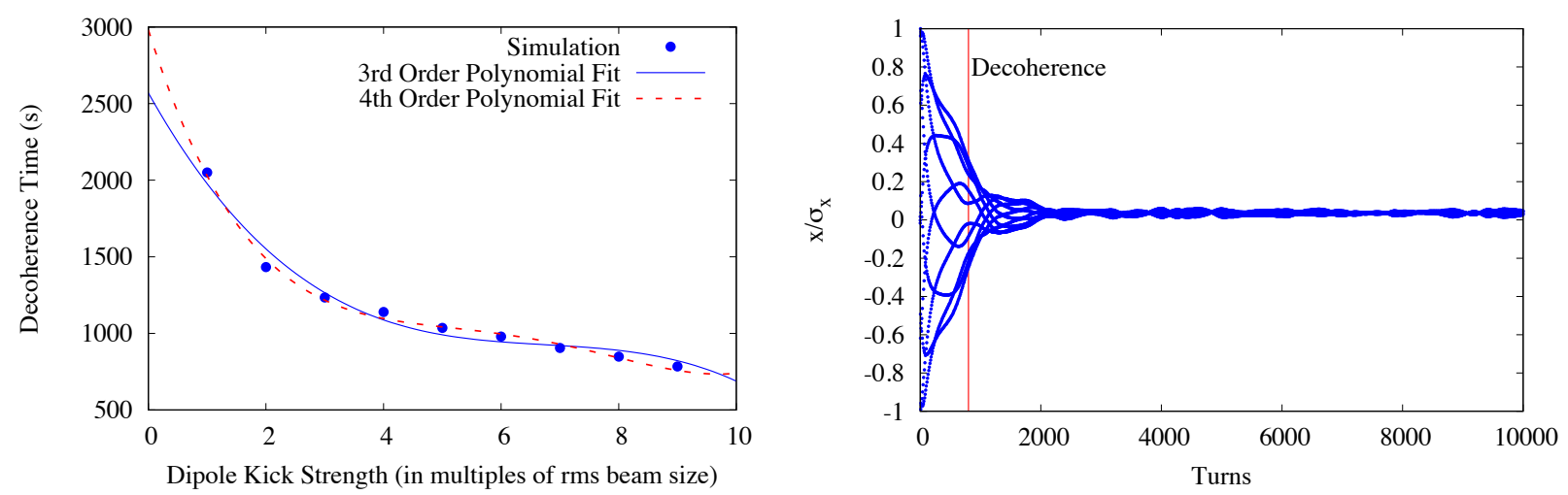

Figure 4: The left is a plot of beam decoherence time versus applied dipole kick strength. The right is a plot of the centroid amplitude as a function of time for the chosen dipole kick strength. The red line shows $t_{D}$, the e-folding time of the centroid amplitude.

These results show an exponential decay relationship between increase in dipole kick strength and decoherence time. Dynamic aperture simulations have shown that the particles 
are stable up to amplitudes of about $25 \times$ rms beam size in the horizontal plane. For all following results a dipole kick of $10 \times$ rms beam size was chosen in order to optimize simulation time and without significant particle losses.

\subsection{Calculation of Optimal Quadruopole Strength}

The nonlinear theory of beam echoes predicts an optimum quadrupole kick using the following equations in the approximation of small amplitude dipole kicks. [6]:

$$
\begin{aligned}
Q & \approx q \tau \omega^{\prime} \epsilon_{0}\left[1+\frac{1}{2}\left(\frac{\beta_{k} \theta}{\sigma_{0}}\right)^{2}\right] \\
\omega^{\prime} & =\nu_{1}-\nu_{o} \\
\langle x(t=2 \tau)\rangle^{a m p} & \approx \beta_{k} \theta \frac{Q}{\left(1+Q^{2}\right)^{3 / 2}} \\
Q_{o p t} & =1 / \sqrt{2} \Rightarrow \frac{\langle x(t=2 \tau)\rangle_{\max }^{a m p}}{\beta_{k} \theta}=0.38
\end{aligned}
$$

where $\mathrm{q}$ is the quadrupole kick strength, $\tau$ is the time period between dipole and quadrupole kicks, $\omega^{\prime}$ is the detuning parameter, $\nu_{o}$ is the tune of the synchronous particle, $\nu_{1}$ is the tune of the $1 \mathrm{rms}$ amplitude particle, $\epsilon_{0}$ is the initial beam emittance, $\beta_{k} \theta$ is the magnitude of the dipole kick, and $\sigma_{0}$ is the initial rms size. These equations can be used to calculate $Q_{o p t}$, the scaled amplitude predicted by the one dimensional nonlinear theory for small amplitude dipole kicks. We use these equations as a rough guide, since the results in [6] for large amplitude dipole kicks, which should be applied here, lead to more complicated equations. The detuning parameter $\left(\omega^{\prime}\right)$ was estimated using the MADX DYNAP module to calculate the tune of particles at a range of amplitudes. The rms tune shift $\left(\nu_{o}-\nu_{1}\right)$ was found to be 0.000011 by calculating the difference in tune between the synchronous particle and a $1 \mathrm{rms}$ amplitude particle, this vaule was used for the detuning parameter. The optimal quadrupole strength "q" was calculated using the above equations. The optimum q was found to be $\mathrm{q}=$ 0.05 for a quadrupole kick applied after a 4000 turn delay time with a dipole kick strength of 10 times the rms beam size. 


\subsubsection{Scan of $q$ values}

In order to test the calculated $q$ value a scan of quadrupole kick values was run for $q=0.01$ 0.1. The amplitude scaled by $\beta_{k} \theta$ of the largest echo produced from these simulations is less than half of that predicted by theory, 0.38. This reduction in amplitude is caused by tranverse coupling and due to nonzero momentum spread of the beam. Theory used to predict 0.38 maximum is valid only for zero momentum spread and without coupling between the transverse planes.
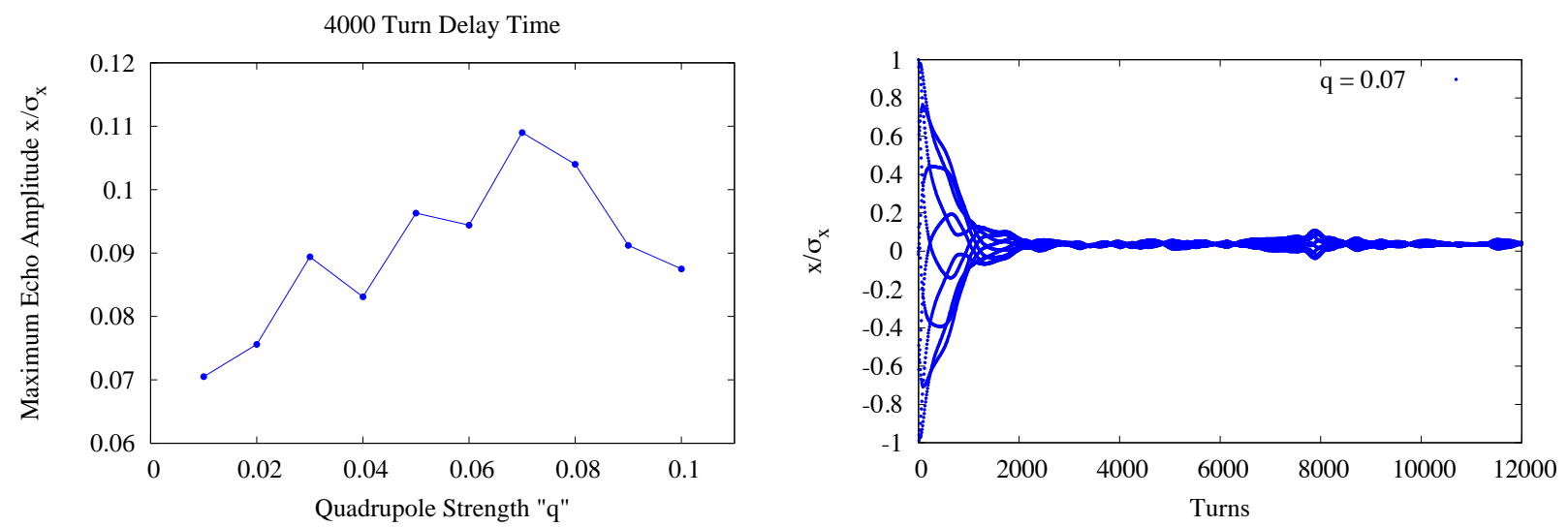

Figure 5: The graph of scaled echo amplitude on the left shows that the optimal quadrupole kick value occurred at $q=0.07$. The graph on the right shows the echo produced by the $\mathrm{q}=0.07$ quadrupole kick.

Echo dependence on quadrupole kick value is expected based on the Nonlinear Theory of Beam Echoes [6]. The discrepancy in the optimum q value predicted by theory is most likely caused by the strong coupling due to the nonzero tune split and non-zero momentum spread. Optimum q value has an inverse relationship to delay time predicted by theory. This optimum q value could then be scaled for different delay times in future simulations.

\subsubsection{Quantum Excitation and Synchrotron Damping}

To test echo dependence on quantum excitation and synchrotron damping simulations were run with these effects removed from the simulation. No noticeable difference in echo amplitude was observed after these settings were removed as shown in 6. This lack of difference is 
because the radiation damping time in IOTA is long so relatively few photons are emitted every turn, so damping and diffusion effects are negligible.
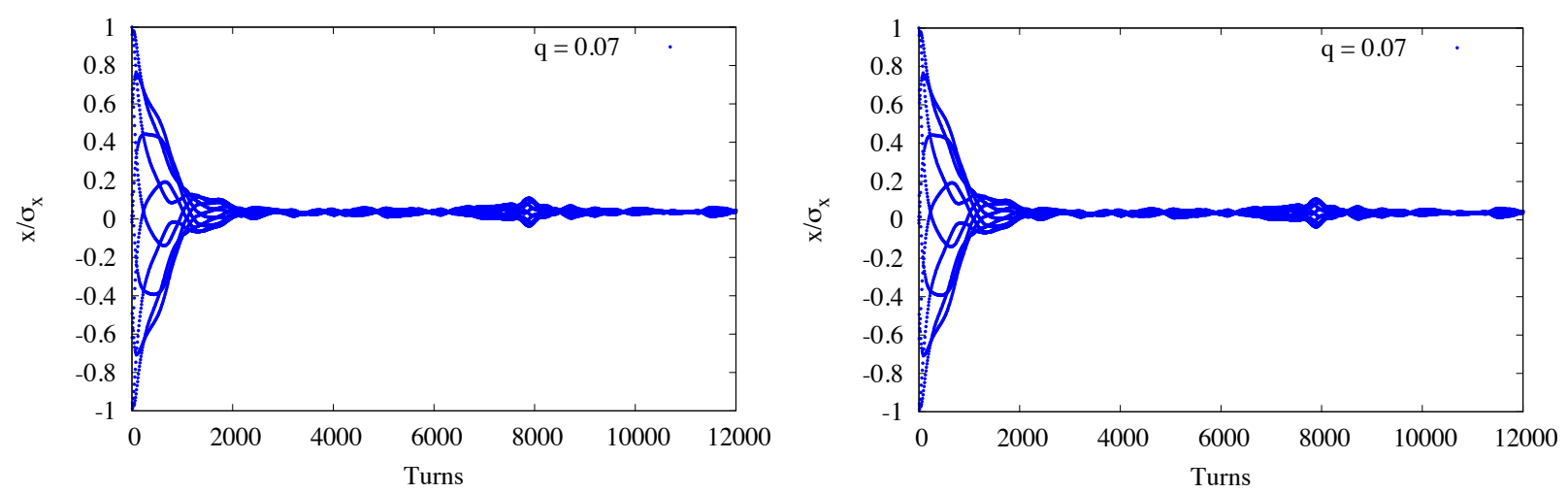

Figure 6: The plot on the left was run with the effects of quantum excitiation and synchrotron damping turned on. The plot on the right was run with the effects of quantum excitation and synchrotron damping removed. Removing quantum excitation and synchrotron damping resulted in no change in the echo effect, as can be seen by these identical plots.

\subsection{Transverse Coupling}

The effects of coupling on the echo amplitude were investigated. Maximum coupling occurs when the $\mathrm{x}$ and $\mathrm{y}$ tunes of the accelerator are equal. When a beam is effected by coupling some of the energy from the dipole kick in the $\mathrm{x}$ plane is transferred to the y plane causing a decrease in particle amplitudes. The strength of coupling can be observed by analyzing the amplitude of the oscillations in the y plane. At greater coupling more energy will be transferred to the y plane yielding higher amplitude oscilla-

Table 1: Optimal q tions, at decreased coupling the opposite effect will occur. The

\begin{tabular}{|c|c|}
\hline$\nu_{y}-\nu_{x}$ & $\mathrm{q}$ \\
\hline-0.0035 & 0.14 \\
0.00 & 0.14 \\
0.0035 & 0.15 \\
0.005 & 0.14 \\
\hline
\end{tabular}
decrease in particle amplitudes in the $\mathrm{x}$ plane caused by coupling then causes a decrease in the overall amplitude of the echo. This effect decreases at increased tune split (difference between the $\mathrm{x}$ and $\mathrm{y}$ tunes).

In order to test the effect of coupling on beam echo amplitude a series of simulations were run with tune splits $\left(\nu_{y}-\nu_{x}\right)=$ 
-0.005 - 0.01. Each tune split was scanned for the optimal quadrupole kick and delay time. Every tune split had an optimal delay time of 2000 turns, but the range tested was small based on previous results. Simulations run after this showed that the optimal delay time was outside of the range tested for these simulations, but the main conclusions remain. Sample optimal quadrupole kick values are shown in Table 1. The lack of deviation from 0.14 indicates that tune split does not have a significant effect on the optimal quadrupole kick strength.

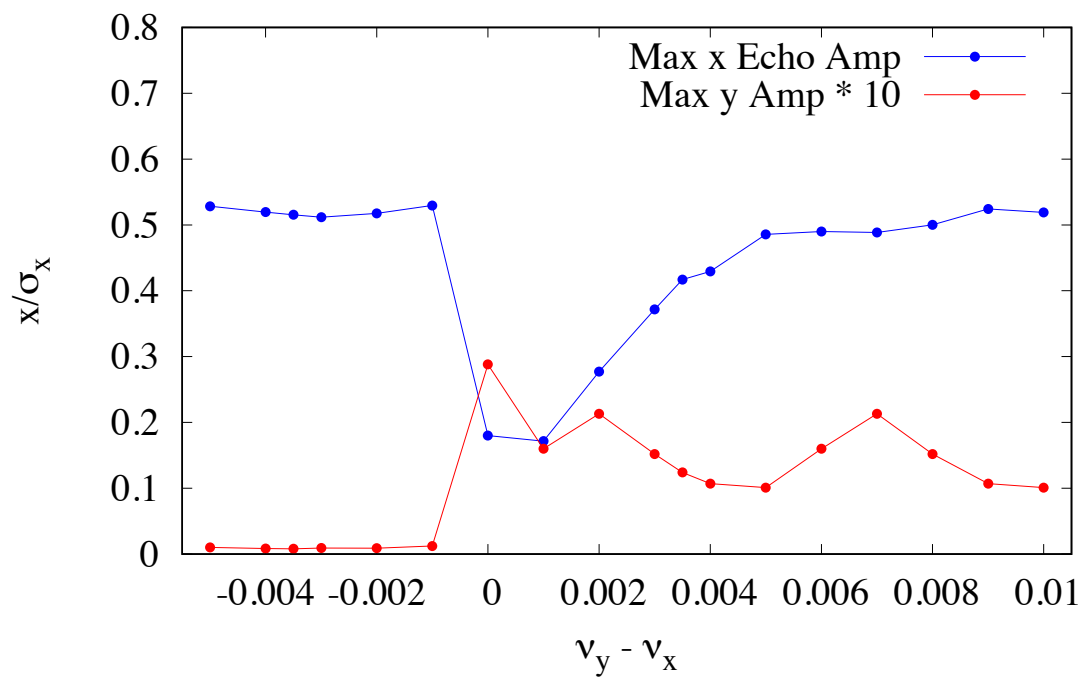

Figure 7: Plot of maximum echo amplitude versus tune split showing significant influence of tune split on echo amplitude.

These results show maximum echo amplitudes that are much greater than the 0.38 predicted by the one dimensional nonlinear theory for small amplitude dipole kicks. They also show an asymmetry where echo amplitudes are larger for $\nu_{x}>\nu_{y}$ than $\nu_{y}>\nu_{x}$ tune split differences when the absolute values of the differences are equivalent. According to the theory for large amplitude dipole kicks, the maximum echo amplitude depends on beam emittance and echo amplitude increases with decreasing beam size. The rms beam size used in these simulations is $0.31 \mathrm{~mm}$ in $\mathrm{x}$ and $0.0425 \mathrm{~mm}$ in $\mathrm{y}$ which is quite small, so echo amplitudes in the range seen here are expected. In Druhv Desai's simulations with protons he found a significant increase in echo amplitude in the 0.00-0.005 tune split range, though the maximum 
echo amplitudes he found where smaller than those found here both results show an upward trend in echo amplitudes with increasing tune split.

These tune split results show that coupling has a significant effect on electron beams and the echoes they are able to produce. The effect of coupling on electron beams can be seen in 8. This graph contains two simulations, one run at maximum coupling (tune split $=0.0$ ) and the other run at one of the smaller coupling values (tune split $=-0.0035$ ). For the maximum coupling result the energy transferred to the y plane causes oscillations that have a large amplitude. For the smaller coupling example the oscillations in the y plane are almost nonexistent due to the lack of energy transfer. Because no energy is transferred to the y plane in the small coupling example all of the energy from the dipole kick can be used to produce a large amplitude echo.

\section{Y Plane Oscillations}

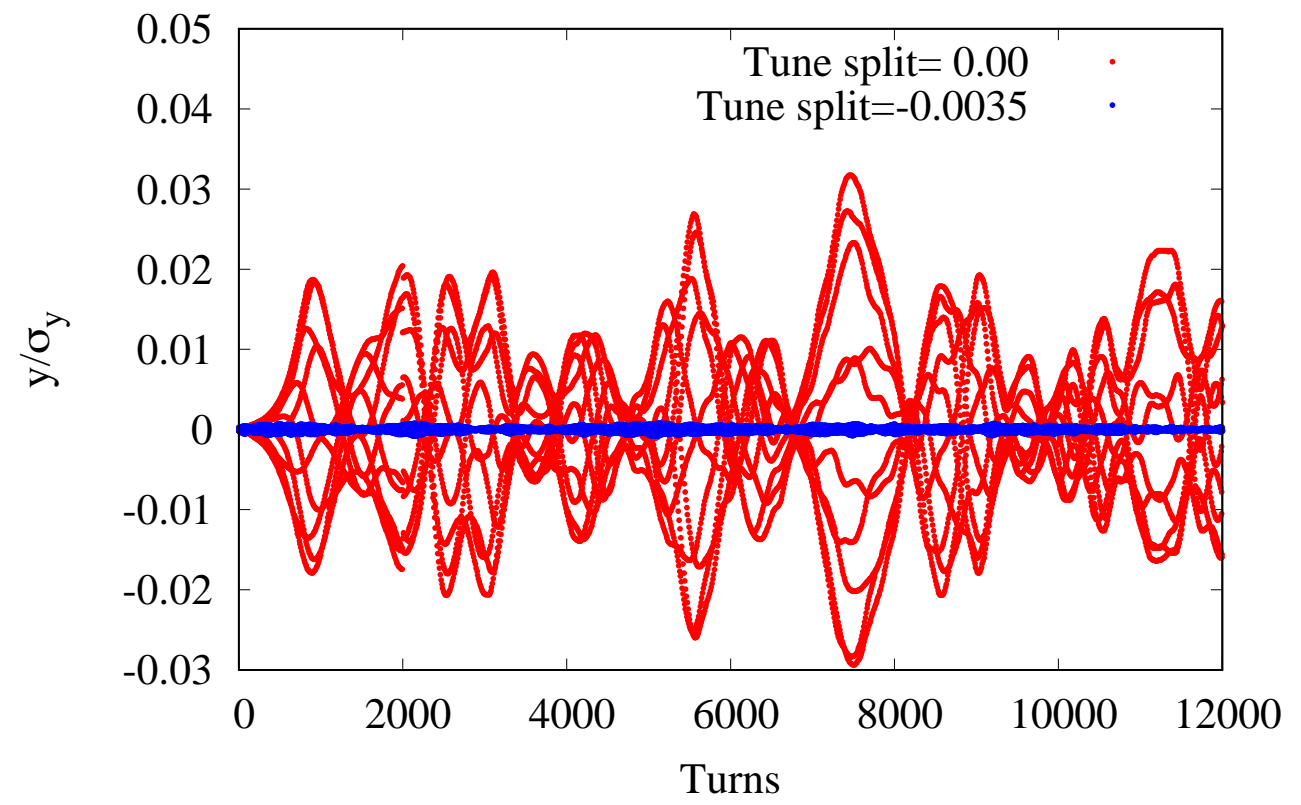

Figure 8: Y plane oscillations for maximum coupling and minimum coupling results.

These results show that the effect of coupling on echo amplitude is very significant and will have to be a carefully controlled parameter in future experiments. According to these results the optimum tune split values for future experiments are $-0.005--0.001$ or 0.008 - 
0.01 .

\subsection{Scan of Delay Times}

In order to determine the optimal delay time a scan of delay times was run with scaled q values. Delays of 1000 - 2000, 2250, and 2500 turns were used. Scans were run at tune splits of -0.0035 small coupling) and 0.00 (maximum coupling). According to current theory optimum quadrupole kick strength scales inversly with delay time, so it was possible to scale the optimum quadrupole kick value found previously for this range of delay times [5]. Echo amplitude peaked at the 1000 turn delay time with $q=0.28$ and tunesplit=-0.0035. An interesting valley was observed where echo amplitudes peaked at 1000 and 2000 turn delay times but drop off in between shown in 8 .

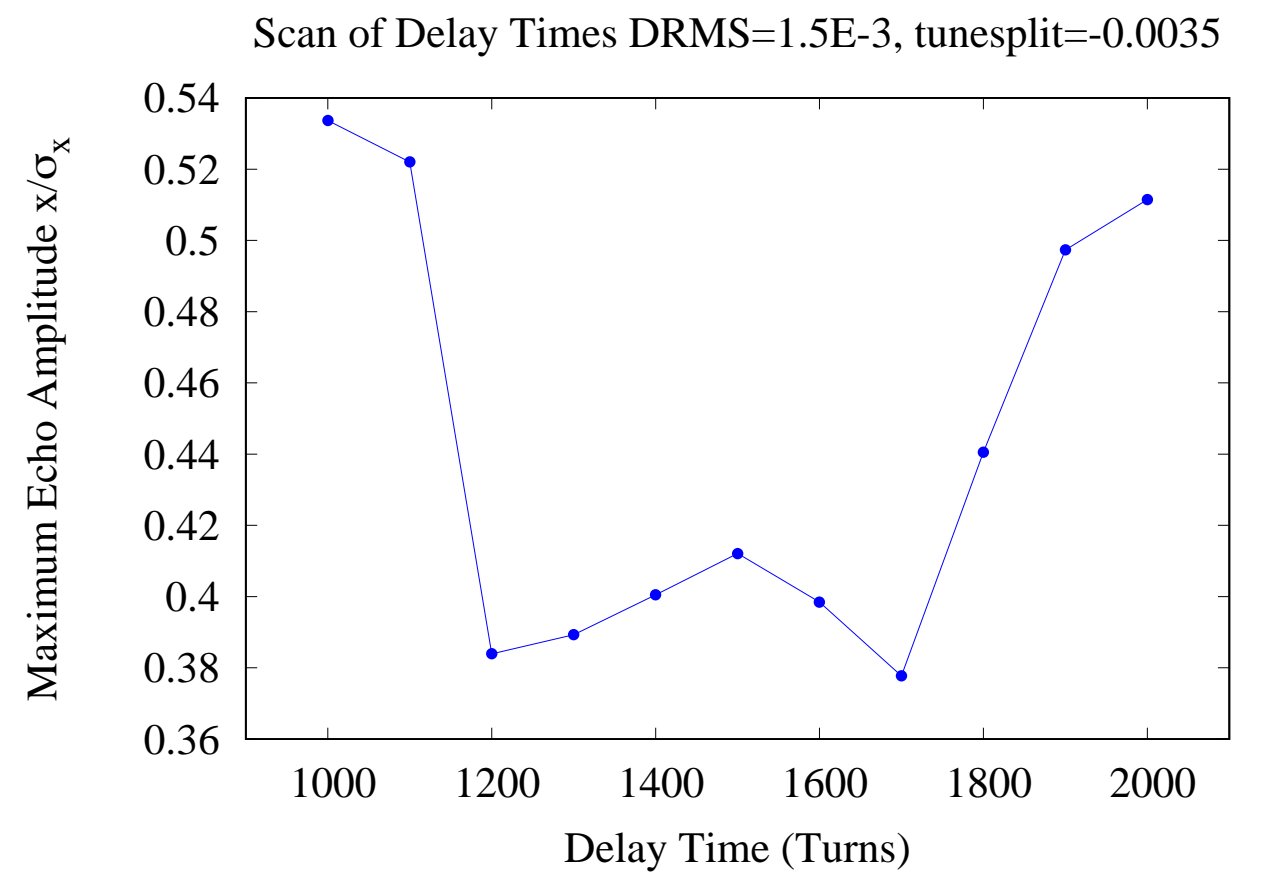

Figure 9: Scan of delay times from 1000 turn to 2000 turn delay time showing maximums at 1000 and 2000 turns. Valley in between 1000 and 2000 turn delay time is also visible. Simulation run with a tune split of -0.0035 and momentum spread of $1.5 \times 10^{-3}$.

Unexpected results were observed at maximum coupling and 1500, 1700, 1800, and 1900 delay times. In these results beam echoes occured immediately before and after the expected 
$2 \tau$ delay times with a minimum at the expected time. Examples of these results are shown in 10. This double echo effect is most likely due to the effects of maximum coupling, double echoes were not observed in the -0.0035 tune split simulations.
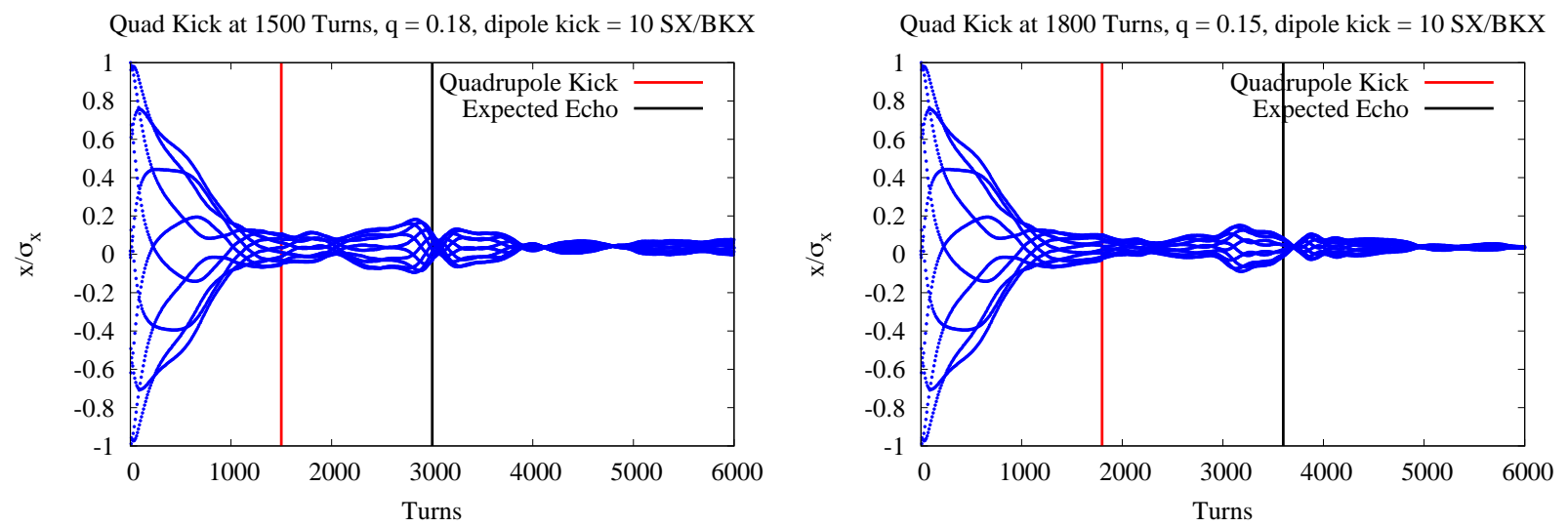

Figure 10: These centroid position graphs demonstrate the unexpected results at 1500 and 1800 turn delay times showing double echo effect. The black "Expected Echo" lines show the expected position of the echo based on theory, it can be seen that for both delay times the echo is actually at a minimum at this point.

Simulations with delay times after 2000 turns showed a decrease in echo amplitude. Echo dependence on delay time is expected based on current theory [5]. These results show optimum echo generation occurs at a 1000 turn delay time with a quadrupole kick value of $\mathrm{q}=0.28$.

\subsection{Momentum Spread}

A scan of different momentum spreads was run in order to determine the effect of momentum spread on maximum echo amplitudes. The tune split was set to -0.0035 as this tune split was in the middle of the maximum echo amplitude tune split range found in the previous results. In order to also determine the effect of momentum spread on quadrupole kick 7 different q values were tried at each delay time for each momentum spread value. 

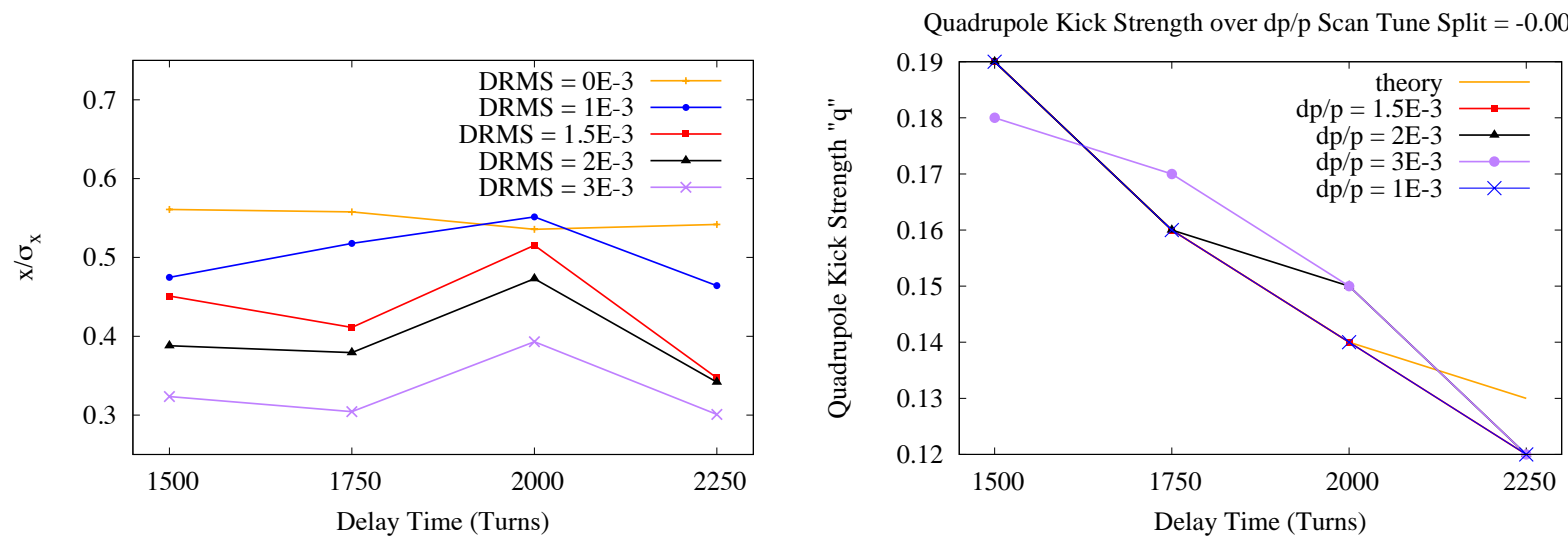

Figure 11: DRMS stands for momentum spread in these plots. The graph at left shows the maximum echo amplitudes achieved at various momentum spreads and delay times. The graph at right shows the ideal $q$ value at different momentum spreads and delay times. The orange line is the ideal q value predicted by theory, it is mostly covered by the black, blue, and red graph lines.

All momentum spread scans display the same general pattern over delay times with an overall decrease in maximum echo amplitude with increased momentum spread. Change in momentum spread did not have a large effect on optimal q value. Ideal q values found in all simulations were within \pm 0.01 of the ideal $q$ value predicted by theory. Changes in momentum spread did not have as large of an effect on echo amplitude as changes in tune split. IOTA has a standard momentum spread of $1.5 \times 10^{-3}$, based on these results it should be possible to generate large amplitude echoes in the IOTA ring.

\subsection{Full Width Half Maximum}

In addition to studies of the effect of various accelerator parameters on echo amplitude we were also interested in the effect of these parameters on the full width half max (FWHM) of the echoes produced. In order to test this simulations used for echo amplitude analysis were analyzed to measure the FWHM of the echoes produced. In these simulations echo FWHM showed a mostly monotonic dependence on the independent variable (the parameter being varied). Results are shown in 12 and 13 . 

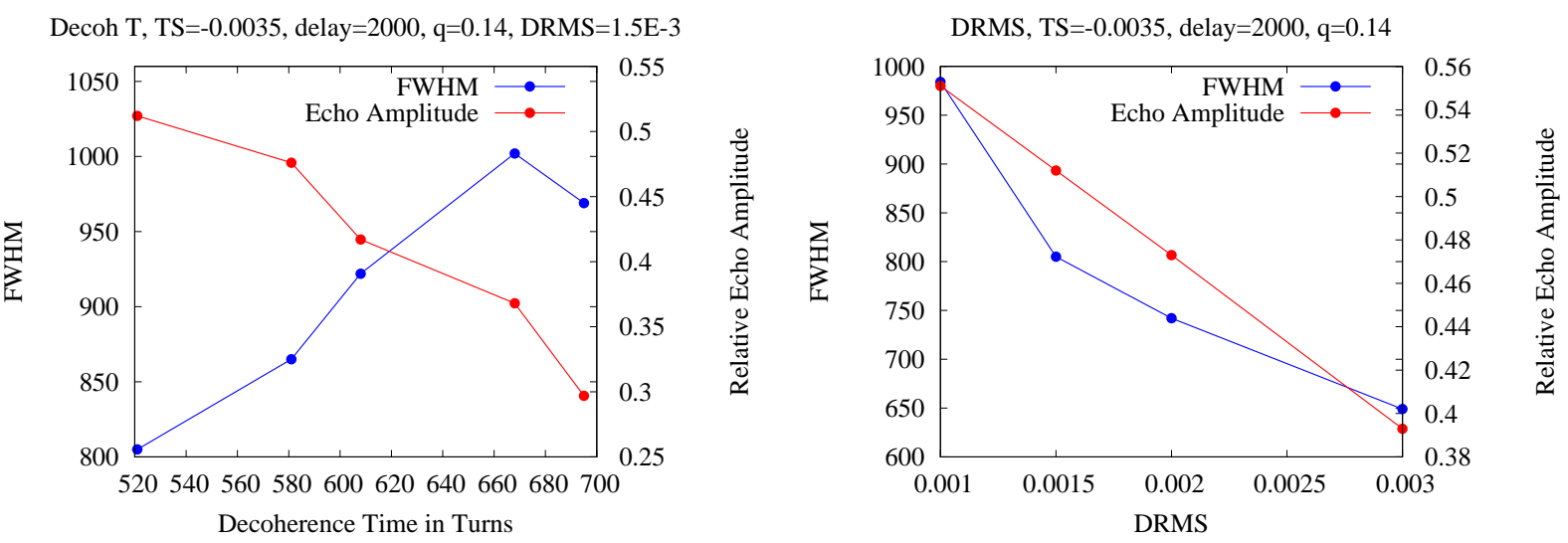

Figure 12: The plot at left shows the relationship between echo FWHM, amplitude, and decoherence time. The plot at right shows the relationship between echo FWHM, amplitude, and momentum spread.
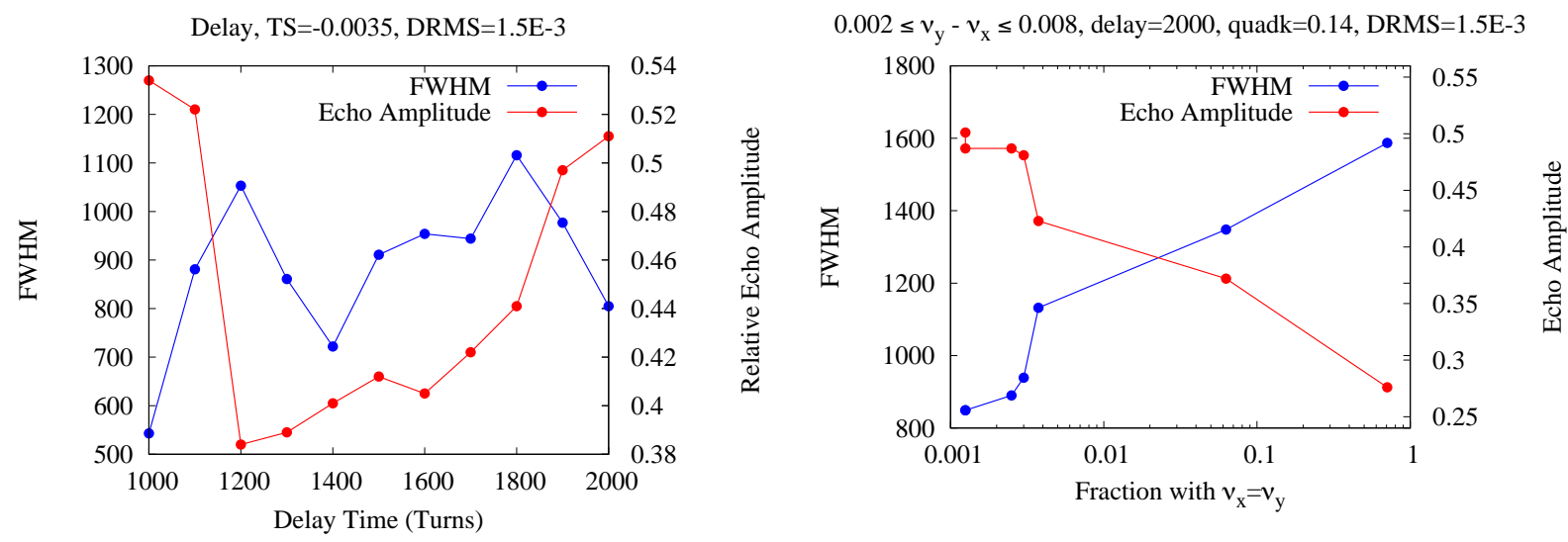

Figure 13: The plot at left shows the relationship between echo FWHM, amplitude, and delay time. The plot at right shows the relationship between echo FWHM, amplitude, and the fraction of particles with the same tune. In the fraction of particles with the same tune plot negative value have been left out in order to make the graph simple to read. In addition results for tune split values of $0.00,0.001$, and 0.009 have been left out due to errors that caused the echo FWHM to be impossible to measure.

In this analysis it was found that FWHM increases with increase in decoherence time. It also decreases with increased momentum spread and fraction of particles with the same tune. In terms of delay time FWHM shows a somewhat oscillatory behavior between delays in the range 1200 - 1800. This oscillatory behavior roughly coincides with the trough in echo amplitudes over the same range. This trough in the echoes and oscillations in the FWHM 
are somewhat unexpected showing that the delay needs to be carefully chosen in future beam echo experiments.

\section{Conclusion}

Through the use of simulations in MADX we have shown that beam echoes can be produced with electrons in the IOTA ring at Fermilab. We find that the synchrotron damping time in IOTA is long enough to allow the generation of significant echoes. Transverse coupling and delay time are shown to have the most significant effect on echo amplitude and full width half max. An interesting asymmetry is observed in the transverse coupling results and the ideal delay time is found to be 1000 turns. These parameters will have to be carefully controlled for in future echo experiments. In other results decoherence time is shown to decrease exponentially with dipole kick and variation in quadrupole kick strength had little effect on echo amplitude. Optimum quadrupole kick strength was found to be close to values predicted by the one dimensional non linear theory for small oscillations. Quantum excitation and synchrotron damping had no effect on echo amplitude due to the long radiation damping time. It was also found that the IOTA momentum spread will be sufficient for echo generation. In order to conduct future experiments at IOTA it will be necessary to re-test these parameters using a smaller dipole kick strength and determine echo robustness to gradient errors.

\section{Acknowledgements}

This research was completed as my senior thesis project at Case Western Reserve University. I would like to thank my mentor Dr.Tanaji Sen for his consistent, patient, and helpful instruction throughout this project. I would also like to thank Professor Michael Martens for his help in getting this project started and and always being willing to answer my questions. Thanks also goes to Professor Rolf Petschek for his assistance through out the year. Finally 
I would like to thank my fellow physics students, friends, and family for being supportive and helpful of all my endeavors.

\section{References}

[1] Chao Alex. Echoes. Lecture Notes at: http://www.slac.stanford.edu/ãchao/lecturenotes.html.

[2] Desai Dhruv. Study of beam echoes in the iota ring, 2018. Final paper written for internship, sent to me by Tanaji Sen.

[3] Grote Hans, Roy Ghislain, and Schmidt Frank. The MAD-X Program (Methodical Accelerator Design) Version 5.04.01 User's Reference Manual. CERN, European Oranisation for Nuclear Research, 2018.

[4] Antipov S, Broemmelsiek D, Bruhwiler D, Edstrom D, Harms E, Lebedev V, Leibfritz J, Nagaitsev S, Park C.s, Piekarz H, Piot P, Prebys E, Romanov A, Ruan J, Sen T, Stancari G, Thangaraj C, Thurman-Keup R, Valishev A, and Shiltsev V. Iota (integrable optics test accelerator): Facility and experimental beam physics program. Journal of Instrumentation, 12:T03002-T03002, 2017.

[5] Sen Tanaji and Fischer Wolfram. Diffusion measurement from observed transverse beam echoes. Physical Review Accelerators and Beams, 20:011001, 2017.

[6] Sen Tanaji and Shen Li Yuan. Nonlinear theory of transverse beam echoes. Physical Review Accelerators and Beams, 21:021002, 2018.

[7] Andy Wolski and David Newton. Design of electron storage and damping rings, 2013. Presentation for the US Particle Accelerator School. 